\title{
Myocardial blood flow: Is motion correction necessary?
}

\author{
Martin Lyngby Lassen, PhD, ${ }^{\mathrm{a}}$ and Piotr J. Slomka, $\mathrm{PhD}^{\mathrm{a}}$ \\ a Artificial Intelligence in Medicine Program, Cedars-Sinai Medical Center, Los Angeles, CA
}

Received Sep 4, 2019; accepted Sep 5, 2019

doi:10.1007/s12350-019-01896-5

\section{See related article, pp. 1334-1346}

Quantification of myocardial blood flow (MBF) with PET has been shown to add prognostic value to myocardial perfusion imaging, ${ }^{1}$ yet some technical aspects of this promising imaging technique have not been fully examined. Like all other PET studies, myocardial studies are affected by the general limitations of the PET systems, including spill-over effects, non-ideal time-of-flight corrections and artifacts in the attenuation correction maps. One of the most critical technical limitations for myocardial perfusion PET is the possible misalignment between the PET emission data and the attenuation correction maps. In several studies, the PET to CT misalignment have been reported to introduce quantitative errors and affect the diagnostic outcome, ${ }^{2,3}$ introducing false-positive findings ${ }^{4-6}$ or even false-negative findings in some cases. ${ }^{2}$ Cardiorespiratory motion during acquisitions is another issue for MBF examinations, where the cardiorespiratory motion might introduce a general smear of the pathologic area. ${ }^{7-9}$ Related to this issue is the wear-off of the pharmaceutical agents used for stress MBF examinations, resulting in changes of the respiratory depth and frequency ${ }^{10-12}$ - a problem often referred to as "myocardial creep". This effect causes repositioning of the heart during scans, and consequently affects the time-activity curves used for the MBF evaluations. ${ }^{13}$

Despite the known problems of the myocardial creep, its impact on the MBF assessments is not yet fully understood. In the current issue of the Journal of

Reprint requests: Martin Lyngby Lassen, PhD, Artificial Intelligence in Medicine Program, Cedars-Sinai Medical Center, 8700 Beverly Blvd. Ste. A047, Los Angeles, CA 90048, USA; martinlyngby.lassen@cshs.org

J Nucl Cardiol 2021;28:1347-8.

$1071-3581 / \$ 34.00$

Copyright (c) 2019 American Society of Nuclear Cardiology.
Nuclear Cardiology, Armstrong et al investigate the impact of the frame-wise motion observed in adenosine ${ }^{82} \mathrm{Rb}$ PET stress scans. ${ }^{14}$ The authors evaluated the impact of frame-by-frame motion correction (MC) on MBF assessments obtained from three different PET image reconstructions using; (a) No MC (standard assessment), (b) MC of PET frames, and (c) synchronized MC of both CTAC images and the reconstructed dynamic PET image series. The patient cohort was divided into three subgroups depending on the motion observed during the scans, ranging from mild to severe. In the study, the authors reported most significant changes in the right coronary artery territory, with median changes in the MBF of $23 \%$ following MC, which is in concordance to previous studies. ${ }^{11,15}$ Despite motion of up to $25 \mathrm{~mm}$ during the scans (myocardial creep), the authors reported that the impact of $\mathrm{MC}$ of the CTAC maps prior to image reconstruction resulted in relatively minor changes of the $\mathrm{MBF}$ (median change $5 \%$ ), compared to the impact of the MC for the dynamic PET image-series alone (median change 12\%). Although the finding is somewhat contradictory to the intuitive logic, similar findings have been reported for ${ }^{82} \mathrm{Rb}$ stress scans using regadenoson. ${ }^{13,16}$

Indeed, the findings reported by Armstrong and Van Dijk $^{14,16}$ are in contrast to the growing evidence that misregistration of the PET and CTAC maps introduce artifacts in the static perfusion images. ${ }^{2,4,5}$ One possible explanation might be related to the stress acquisition protocols. The recent joint position paper from the SNMMI Cardiovascular Council and ASNC proposes that stress MPI scans are initiated either by mid-infusion of the stress agent when using adenosine (infusion duration 4-6 minutes) or immediately after injection of $10 \mathrm{~mL}$ saline when using regadenoson. ${ }^{1}$ Owing to the biological half-lives of the vasodilators (Adenosine $<10$ seconds, Regadenoson 2-3 minutes $^{10}$ ), the patient will be experiencing full vasodilation in the first 120 seconds of the acquisition. Several studies have shown that MC of this time period alone might be sufficient to obtain 
reproducible MBF assessments. ${ }^{15,17}$ The consistent translation of the myocardium during the first $120 \mathrm{sec}$ onds reported by Armstrong et $\mathrm{al}^{14}$ might be the reason that the impact of synchronous MC of PET and CTAC maps is suppressed when compared to MC of the dynamic PET images alone.

Does this mean that MC of the CTAC map should be completely omitted in the future? Most likely not, as current MBF estimates focus on dynamic reconstructions without corrections for cardiorespiratory motion, which still might introduce significant changes in to the MBF evaluations. ${ }^{18}$ The inclusion of cardiorespiratory $\mathrm{MC}$ in the clinical practice would require highly specialized reconstruction protocols, in which the corrections are introduced during the PET image reconstructions. Such protocols could be expanded to also include synchronized corrections for both myocardial creep and MC of the CTAC maps. However, because of their complexity, it will not be possible to introduce these reconstruction protocols to the clinical practice without support from the vendors.

\section{References}

1. Murthy VL, Bateman TM, Beanlands RS, et al. Clinical quantification of myocardial blood flow using PET: Joint position paper of the SNMMI cardiovascular council and the ASNC. J Nucl Med 2018;59:273-93. https://doi.org/10.2967/jnumed.117.201368.

2. Slomka PJ, Diaz-Zamudio M, Dey D, et al. Automatic registration of misaligned $\mathrm{CT}$ attenuation correction maps in Rb-82 PET/CT improves detection of angiographically significant coronary artery disease. J Nucl Cardiol 2015;22:1285-95. https://doi.org/10.1007/ s12350-014-0060-9.

3. Pan T, Mawlawi O, Luo D, et al. Attenuation correction of PET cardiac data with low-dose average CT in PET/CT. Med Phys 2006;33:3931-8. https://doi.org/10.1118/1.2349843.

4. Martinez-Möller A, Souvatzoglou M, Navab N, et al. Artifacts from misaligned CT in cardiac perfusion solutions. J Nucl Med 2007;48:188-94.

5. Gould KL, Pan T, Loghin C, et al. Frequent diagnostic errors in cardiac PET/CT due to misregistration of CT attenuation and emission PET images: A definitive analysis of causes, consequences, and corrections. J Nucl Med 2007;48:1112-21. https://d oi.org/10.2967/jnumed.107.039792.

6. Sureshbabu W, Mawlawi O. PET/CT imaging artifacts. J Nucl Med Technol 2005;33:156-61.
7. Lassen ML, Beyer T, Berger A, et al. Data-driven, projectionbased respiratory motion compensation of PET data for cardiac PET/CT and PET/MR imaging. J Nucl Cardiol 2019. https://doi. org/10.1007/s12350-019-01613-2.

8. Gigengack F, Ruthotto L, Burger M, et al. Motion correction in dual gated cardiac PET using mass-preserving image registration. IEEE Trans Med Imaging 2012;31:698-712.

9. Wang X, Rahmim A, Tang J. MRI assisted dual motion correction for myocardial perfusion defect detection in PET imaging. Med Phys 2017;44:4536-47.

10. Vleeming EJ, Lazarenko SV, van der Zant FM, et al. Cardiac displacement during $13 \mathrm{n}$-ammonia myocardial perfusion pet/ct: Comparison between adenosine- and regadenoson-induced stress. J Nucl Med Technol 2018;46:114-22. https://doi.org/10.2967/jnm t.117.199463.

11. Koenders SS, van Dijk JD, Jager PL, et al. How to detect and correct myocardial creep in myocardial perfusion imaging using Rubidium-82 PET? J Nucl Cardiol 2019;26:729-34. https://doi. org/10.1007/s12350-019-01650-X.

12. Memmott MJ, Tonge CM, Saint KJ, Arumugam P. Impact of pharmacological stress agent on patient motion during rubidium82 myocardial perfusion PET/CT. J Nucl Cardiol 2018;25:128695. https://doi.org/10.1007/s12350-016-0767-x.

13. Hunter CRRN, Klein R, Beanlands RS, DeKemp RA. Patient motion effects on the quantification of regional myocardial blood flow with dynamic PET imaging. Med Phys 2016;43:1829. http s://doi.org/10.1118/1.4943565.

14. Armstrong IS, Memmott MJ, Saint KJ, et al. Assessment of motion correction in dynamic rubidium-82 cardiac PET with and without frame-by-frame adjustment of attenuation maps for calculation of myocardial blood flow. J Nucl Cardiol 2019. https://doi.org/10. 1007/s12350-019-01834-5.

15. Lee BC, Moody JB, Poitrasson-Rivière A, et al. Blood pool and tissue phase patient motion effects on 82rubidium PET myocardial blood flow quantification. J Nucl Cardiol 2018. https://doi.org/10. 1007/s12350-018-1256-1.

16. van Dijk JD, Jager PL, Ottervanger JP, et al. No need for framewise attenuation correction in dynamic Rubidium-82 PET for myocardial blood flow quantification. J Nucl Cardiol 2019;26:73845. https://doi.org/10.1007/s12350-019-01654-7.

17. Otaki Y, Lassen ML, Manabe O, et al. Short-term repeatability of myocardial blood flow using 82Rb PET/CT: the effect of arterial input function position and motion correction. J Nucl Cardiol 2019. https://doi.org/10.1007/s12350-019-01888-5.

18. Germino M, Carson RE. Cardiac-gated parametric images from 82Rb PET from dynamic frames and direct 4D reconstruction. Med Phys 2018;45:639-54

Publisher's Note Springer Nature remains neutral with regard to jurisdictional claims in published maps and institutional affiliations. 